\title{
COMPLEXES AND MANIFOLDS REPRESENTED BY FUNCTIONS OF REAL VARIABLES*
}

\author{
BY W. M. WHYBURN
}

It is the purpose of this paper to show that a wide class of loci in a real space of $n$ dimensions is composed of sets which are complexes and manifolds in the sense of combinatorial analysis situs. The combinatorial approach to analysis raises the important question of determining the conditions that a real function $u=f\left(x_{1}, \cdots, x_{n}\right)$ of a finite set of real variables must satisfy in order that this function generate a locus which can be identified as a cell, complex, or manifold. $\dagger$ Answers to this question form essential links between combinatorial analysis situs and real variable theory. Van der Waerden, $\ddagger$ Lefschetz, $\S$ Koopman and Brown, $\|$ have studied this question for cases where the function $u$ is analytic. S. S. Cairns $\uparrow$ has investigated the cases that arise for functions $u$ that are continuous together with their first partial derivatives. The present paper deals with functions $u$ which are continuous but which are not required to have first partial derivatives at any points of their domains of definition. The restrictions placed on the functions are essentially those used by Hedrick and Westfall ${ }^{* *}$ in their general

* Presented to the Society, November 30, 1935.

$\dagger$ See Veblen, Analysis Situs, Colloquium Publications of this Society, vol. 5 , part 2 .

¥ Mathematische Annalen, vol. 102 (1929), pp. 360-361.

$\S$ Topology, Colloquium Publications of this Society, vol. 12, chapter 8 . On page 364, Lefschetz expressed a belief that van der Waerden (loc. cit.) was first to establish connections between functionally defined loci and complexes. It should be mentioned that a slightly earlier paper by W. M. Whyburn [this Bulletin, vol. 35 (1929), p. 706] contained a proof that a locus of a general type was a manifold and also carried a reference to results of this type which S. S. Cairns had obtained but had not published.

\| Transactions of this Society, vol. 34 (1932), pp. 231-251.

I See his papers in Annals of Mathematics, (2), vol. 35 (1934), pp. 579-587, and this Bulletin, vol. 41 (1935), pp. 549-552, where references to his earlier work will be found.

** Bulletin de la Société Mathématique, vol. 44, pp. 1-14, and Festschrift David Hilbert, Berlin, 1922, pp. 74-77, or Mathematische Annalen, vol. 85 (1922), pp. 74-77. 
implicit function theorem. The use of this latter theorem makes it possible for proofs to be given without the use of partial derivatives, directional derivatives, or direction cosines. This omission of derivatives is desirable since derivatives do not have a role in analysis situs. Continuity, on the other hand, enters into the transformations and correspondences of analysis situs.

Theorem 1. Let $H: A_{i} \leqq x_{i} \leqq B_{i},(i=1,2, \cdots, n)$, be the interior and boundary of an n-rectangle in the space of the $n$ real variables $x_{1}, x_{2}, \cdots, x_{n}$, and let $K$ be a closed connected subset of $H$. For each point $P$ of $K$, let there exist an n-rectangular neighborhood of $P$, $H_{P}: A_{P i} \leqq x_{i} \leqq B_{P i},(i=1, \cdots, n)$, such that the subset of $K$ that belongs to $H_{P}$ is identical with the set of points which satisfies a single equation $x_{j}=f\left(x_{1}, \cdots, x_{j-1}, x_{j+1}, \cdots, x_{n}\right)$, where $f$ is continuous and $A_{P j}<f<B_{P j}$ on the set of points $A_{P i} \leqq x_{i} \leqq B_{P i}$, $(i=1,2, \cdots, j-1, j+1, \cdots, n)$. Under these hypotheses, the point set $K$ is an $(n-1)$-complex.*

Proof. Let $\left[H_{P}\right]$ be the collection of rectangles obtained when each point $P$ of $K$ is covered as indicated in the hypotheses of the theorem. Since $K$ is closed and bounded, the HeineBorel theorem may be applied to get a finite sub-collection of this collection which covers $K$. Let such a finite sub-collection be chosen and let $H_{1}, H_{2}, \cdots, H_{m}$ be the finite collection of $n$ polygons formed from this collection when each pair of rectangles of the collection that have interior points in common, and that have the further property that the subsets of $K$ belonging to them are represented by functions of the same $n-1$ variables, is replaced by their sum and this process is continued until no two of the sets $H_{1}, \cdots, H_{m}$ which have interior points in common have the same $n-1$ variables of the set $\left(x_{1}, \cdots, x_{n}\right)$ as independent variables in the functions that represent the subsets of $K$ that they contain. Let $H_{j}^{\prime}$ be the boundary of $H_{j}$ and $p$ be a point of $K$ that belongs to $H-\left(H^{\prime}+H_{1}^{\prime}+\cdots+H_{m}^{\prime}\right)$. Let $h_{p}$ be the maximal connected subset of $H-\left(H^{\prime}+H_{1}^{\prime}+\cdots+H_{m}{ }^{\prime}\right)$ that contains $p$, together with the boundary of this maximal connected subset. If $\left[h_{p}\right]$ denotes the collection of sets obtained in this way, then $\left[h_{p}\right]$ consists of a finite set, $h_{1}, h_{2}, \cdots, h_{q}$, of

* The terms "complex," "cell," "circuit," "manifold," . . , are used with the meanings given to them in Veblen's book, loc. cit. 
$n$-polygons (interiors and boundaries) each of which has its face $(n-1)$-polygons subsets of the faces of $H, H_{1}, \cdots, H_{m}$.

Lemma 1. Every point of $K$ is a limit point of a subset of $K$ that is interior to some one of the polygons $h_{1}, h_{2}, \cdots, h_{q}$.

Proof of Lemma 1 . The connectivity of $K$ shows that any point of $K$ which is interior to one of the sets $h_{1}, \cdots, h_{q}$, is a limit point of points of $K$ interior to this. If $p:\left(a_{1}, a_{2}, \cdots, a_{n}\right)$ is a point of $K$ not interior to any $h_{j}, p$ is interior to some $H_{r}$ of the set $H_{1}, \cdots, H_{m}$, and, with a suitable permutation of the subscripts of the $x$ 's, the points of $K$ that belong to $H_{r}$ are solutions of the equation $x_{1}=f\left(x_{2}, \cdots, x_{n}\right)$. We may therefore pick a sequence of points $p_{i}:\left(a_{i 1}, \cdots, a_{i n}\right),(i=1,2, \cdots)$, of $K$ with $p$ as its sequential limit point and such that $a_{i 2} \neq a_{2}$, $a_{i 3} \neq a_{3}, \cdots, a_{i n} \neq a_{n},(i=1,2, \cdots)$. If $x=a_{1}$ is not a face that contains $p$ and belongs to any polygon of the set $h_{1}, \cdots, h_{q}$, then infinitely many of the points $p_{i}$ are interior to some one of the finite set $h_{1}, \cdots, h_{q}$ and $p$ is on the boundary of this set. If $x=a_{1}$ is a face that contains $p$ and belongs to one of the $h$ 's, then this face belongs to one of the polygons $H_{r}, s \neq r$, in which an equation $x_{2}=g\left(x_{1}, x_{2}, \cdots, x_{n}\right)$ holds (after a suitable choice of subscripts on the $x$ 's). If we make use of the single-valuedness and continuity of $f$ and $g$, we are able to choose the points $p_{i}$ so that they have the additional property $a_{i 1} \neq a_{1},(i=1,2, \cdots)$. With this choice of the points $p_{1}, p_{2}, \cdots$, only a finite number of them will lie on faces of the polygons $h_{1}, \cdots, h_{q}$ and hence infinitely many of them will be interior to some one of this finite set of polygons and $p$ will be on the boundary of such a polygon. This completes the proof of the lemma.

LEMma 2. Let $K_{i}$ be the subset of $K$ that is on the boundary of $h_{i}$ and is such that each point of $K_{i}$ is a limit point of points of $K$ that are interior to $h_{i}$. The set $K_{i}$ is a finite collection of $(n-2)$ circuits each of which is the boundary of an $(n-1)$-cell that is a subset of $K$ and is interior to $h_{i}$.

Proof of Lemma 2. We use mathematical induction. The case $n=2$ yields a set of plane polygons for $h_{1}, h_{2}, \cdots, h_{q}$, with faces parallel to the coordinate axes. Let $h_{i}$ be a subset of $H_{r}$ and let $x_{2}=f\left(x_{1}\right)$ be the equation associated with $H_{r}$. Since this equation is satisfied by the subset of $K$ in $h_{i}$, it follows that $K_{i}$ 
has at most one point, $[c, f(c)]$, in any face $x_{1}=c$ of $h_{i}$. If $h_{i}$ has a face $x_{2}=c$ which contains a point of $K_{i}$, then such a face must belong to a polygon of the set $H_{1}, \cdots, H_{m}$ in which an equation $x_{1}=g\left(x_{2}\right)$ holds, since $H_{r}$ has no face of the form $x_{2}=$ constant which contains a point of $K$. The equation $x_{1}=g\left(x_{2}\right)$ yields at most one point of $K_{i}$ on this face. The set $K_{i}$ consists of a finite number of zero cells and the single-valuedness of $f$ and $g$ shows that each of these can be on the boundary of at most two maximal connected subsets of $K$ that belong to the interior of $h_{i}$. If $k$ is a maximal connected subset of $K$ that is interior to $h_{i}$, the equation $x_{2}=f\left(x_{1}\right)$ establishes a one-to-one continuous correspondence between $k_{1}$ and a closed interval on the $x_{1}$ axis, where $k_{1}$ is $k$ together with its boundary. The set $k_{1}$ is therefore an $(n-1)$-complex consisting of an $(n-1)$-cell interior to $h_{i}$ and its $(n-2)$-circuit boundary composed of two of the zero cells of $K_{i}$. Since each point of $K_{i}$ is on the boundary of at least one set of type $k$ and is not on the boundary of more than two such sets, it follows that the lemma is true for the case $n=2$.

Let the lemma hold up to and including the case $n-1$. In the case of $n$ variables, let $h_{i}$ be one of the polygons of the set $h_{1}, \cdots, h_{q}$ and let $H_{r}$ be a polygon of the set $H_{1}, \cdots, H_{m}$ that contains $h_{i}$. Let the equation $x_{1}=f\left(x_{2}, \cdots, x_{n}\right)$ hold for the subset of $K$ that belongs to $H_{r}$. The subset of $K_{i}$ that belongs to any face of the form $x_{j}=c,(j=2, \cdots, n)$, satisfies the equation $x_{1}=f\left(x_{2}, \cdots, x_{j-1}, c, x_{j+1}, \cdots, x_{n}\right)=F\left(x_{2}, \cdots, x_{j-1}, x_{j+1}, \cdots, x_{n}\right)$. The single-valuedness of $F$ together with an application of the lemma for the case $n-1$ shows that this intersection consists of a finite number of $(n-2)$-cells and their bounding $(n-3)$-circuits. If $K_{i}$ intersects a face of the type $x_{1}=c$, where $c$ is a constant, then such an intersection satisfies an equation of the form $x_{2}=g\left(x_{1}, x_{3}, \cdots, x_{n}\right)$ (since such a face must belong to some $H_{j}$ other than $H_{r}$ ). The single-valuedness of $g$ together with an application of the lemma for $n-1$ shows that this intersection is a finite set of $(n-2)$-cells together with their bounding $(n-2)$-circuits. Let $k$ be a maximal connected subset of $K$ that is interior to $h_{i}$ and let $k_{1}$ denote $k$ together with its boundary. The equation $x_{1}=f\left(x_{2}, \cdots, x_{n}\right)$ establishes a oneto-one continuous correspondence between $k_{1}$ and an $(n-1)$ complex [composed of an $(n-2)$-circuit and the $(n-1)$-cell that it bounds] in the hyperplane $x_{1}=0$. Hence $k_{1}$ is an $(n-1)$ - 
complex consisting of an $(n-1)$-cell and its bounding $(n-2)$ circuit. The lemma follows at once when it is noted that each point of $K_{i}$ is on the boundary of at least one maximal connected subset of type $k$ and (from the single-valuedness of the functions $f$ and $g$ ) no such point is on the boundary of more than two sets of type $k$.

Proof of Theorem 1. The theorem follows at once from the lemmas. The set $K$ is an $(n-1)$-complex composed of the $(n-1)$-cells of Lemma 2 together with their boundaries which are the $(n-2)$-circuits described in that lemma. Lemma 1 shows that all of $K$ belongs to this complex, while the fact that the $(n-1)$-cells are mutually exclusive follows from the observation that no two of the sets $h_{1}, \cdots, h_{q}$ have interior points in common. A subdivision of the $(n-2)$-circuits of this complex into cells of orders $n-2, n-3, \cdots, 0$ is obtained when the reasoning used in the proof of Lemma 2 is repeated, with slight verbal modifications, for the $(n-1)$-polygons obtained on the faces of $h_{1}, \cdots, h_{q}$.

TheOREM 2. Under the hypotheses of Theorem 1, if the set $K$ is interior to $H, K$ is a closed ( $n-1)$-manifold.

Proof. From Theorem $1, K$ is an $(n-1)$-complex. Since $K$ is interior to $H$, we may make the neighborhood $H_{P}$ of Theorem 1 so that it is interior to $H$. The equation $x_{j}=\left(x_{1}, \cdots, x_{j-1}\right.$, $\left.x_{j+1}, \cdots, x_{n}\right)$ establishes a one-to-one continuous correspondence between the subset of $K$ in $H_{P}$ and the interior and boundary of an $(n-1)$-rectangle in the hyperplane $x_{j}=0$. Hence the subset of $K$ in $H_{P}$ is an $(n-1)$-cell and boundary and thus each point of $K$ has a neighborhood which is an $(n-1)$-cell. Hence $K$ is a closed $(n-1)$-manifold.*

Corollary. If $K$ is interior to $H$ and the hypotheses of Theorem 1 are satisfied, $K$ is an $(n-1)$-circuit that forms the boundary of an $n$-cell interior to $H$.

Theorem 3. Let the real function $f\left(x_{1}, \cdots, x_{n}\right)$ be contin-

* This theorem would also follow as a corollary to the proof of Theorem 1 if it were observed that this proof shows each $(n-2)$-cell of the complex $K$ to be on the boundary of at least one but not more than two $(n-1)$-cells. When $K$ is interior to $H$, the single-valued functions that define $K$ in a neighborhood of any point $p$ make each $(n-2)$-cell incident with exactly two $(n-1)$-cells. 
uous in an open portion $D$ of the space of the $n$ real variables $\left(x_{1}, \cdots, x_{n}\right)$. Let $H$ be an $n$-rectangle together with its interior which is a subset of $D$ and let $K$ be the points of $H$ which satisfy $f\left(x_{1}, \cdots, x_{n}\right)=0$. For each point $p$ of $K$, let there exist an $n$ rectangular neighborhood throughout which some one of the difference quotients $a_{j},(j=1,2, \cdots, n)$, is definite in sign, where

$$
a_{j}=\frac{f\left(x_{1}, \cdots, x_{j-1}, x_{j}^{\prime}, x_{j+1}, \cdots, x_{n}\right)-f\left(x_{1}, \cdots, x_{n}\right)}{x_{j}^{\prime}-x_{j}} .
$$

Under these hypotheses, the set $K$ is an $(n-1)$-complex. Furthermore, if $K$ is interior to $H, K$ is a finite set of closed $(n-1)$ manifolds.

Proof. This theorem is an immediate consequence of Theorems 1 and 2 when account is taken of an implicit function theorem by Hedrick and Westfall.* This implicit function theorem applies directly to show that the hypotheses of Theorem 1 are satisfied for each connected subset of $K$. The same implicit function theorem shows that $K$ consists of a finite number of maximal connected subsets. The latter fact follows from the observation that the sum of infinitely many such subsets would have a limit point in $K$ and the uniqueness part of the implicit function theorem would break down at such a limit point. The conclusions of Theorem 3 follow from the conclusions of Theorems 1 and 2 .

Theorem 4. Let $H: A_{i} \leqq x_{i} \leqq B_{i},(i=1,2, \cdots, n)$, be the interior and boundary of an n-rectangle in the space of the $n$ real variables $\left(x_{1}, \cdots, x_{n}\right)$ and let $K$ be a closed, connected subset of $H$. For each point $p$ of $H$, let there exist an n-rectangular neighborhood $H_{p}: A_{p i} \leqq x_{i} \leqq B_{p i},(i=1, \cdots, n)$, of $p$ such that the subset of $K$ that belongs to $H_{p}$ is identical with the set of points which satisfies a unique system of $r$ equations:

$$
\begin{gathered}
x_{j_{1}}=g_{1}\left(x_{j_{r+1}}, \cdots, x_{j_{n}}\right), \quad x_{j_{2}}=g_{2}\left(x_{j_{r+1}}, \cdots, x_{j_{n}}\right), \\
\cdots, \quad x_{j_{r}}=g_{r}\left(x_{j_{r+1}}, \cdots, x_{j_{n}}\right),
\end{gathered}
$$

where $j_{1}, \cdots, j_{n}$ is an arrangement of the indices $1,2, \cdots, n$ that is fixed for each $H_{p}$, and where the functions $g_{1}, \cdots, g_{r}$ are

* Loc. cit. 
continuous and satisfy $A_{p j_{1}}<g_{1}<B_{p j_{1}}, \cdots, A_{p j_{r}}<g_{r}<B_{p j_{r}}$ on the point set $A_{p i} \leqq x_{i} \leqq B_{p i}, \quad\left(i=j_{r+1}, \cdots, j_{n}\right)$. Under these hypotheses, the point set $K$ is an $(n-r)$-complex.

Proof. Let $n$-polygons $h_{1}, \cdots, h_{q}$ be constructed as they were in the proof of Theorem 1 (with the variation that the role of the single function $f$ of Theorem 1 is now played by the $r$ functions $\left.g_{1}, \cdots, g_{n}\right)$. Consider the subset $k$ of $K$ that belongs to $h_{i}$ and let the notation be chosen so that $x_{1}=g_{1}\left(x_{r+1}, \cdots, x_{n}\right), \cdots$, $x_{r}=g_{r}\left(x_{r+1}, \cdots, x_{n}\right)$ are the equations satisfied by the points of $k$. The point set $k$ is in one-to-one continuous correspondence with $a$ set of points $k^{\prime}$ in the $n-r+1$ plane: $x_{2}=0, \cdots, x_{r}=0$ if we let the point $P\left(x_{1}, x_{2}, \cdots, x_{r}, x_{r+1}, \cdots, x_{n}\right)$ of $k$ correspond to the point $P^{\prime}\left(x_{1}, 0,0, \cdots, 0, x_{r+1}, \cdots, x_{n}\right)$ of $k^{\prime}$. Furthermore, the set $k^{\prime}$ satisfies the equation $x_{1}=g_{1}\left(x_{r+1}, \cdots, x_{n}\right)$. An application of Theorem 1 for the case $n-r+1$ shows that $k^{\prime}$ is an $(n-r)$-complex. Since $k$ is in one-to-one continuous correspondence with $k^{\prime}$, it follows that $k$ is also an $(n-r)$-complex. An application of this same type of reasoning shows that the subset of $k$ that belongs to any face of $h_{i}$ as well as the subset of $k$ that is common to a face of $h_{i}$ and a face of $h_{j}, j \neq i$, is an $(n-r-1)$-complex. It follows that $K$ is an $(n-r)$-complex with its $(n-r)$-cells interior to the polygons $h_{1}, \cdots, h_{q}$ and that the $(n-r-1)$-circuits that bound these cells lie in the boundaries of these polygons.

An adaptation of the proof of Theorem 2 yields the following theorem.

THEOREM 5. Under the hypotheses of Theorem 4, if the set $K$ is interior to the rectangle $H$, then $K$ is a closed $(n-r)$-manifold.

THEOREM 6. Let the real functions $f_{1}\left(x_{1}, \cdots, x_{n}\right), \cdots$, $f_{r}\left(x_{1}, \cdots, x_{n}\right)$ be continuous in an open portion $D$ of the space of the $n$ real variables $\left(x_{1}, \cdots, x_{n}\right)$. Let $H: A_{i} \leqq x_{i} \leqq B_{i},(i=1, \cdots$, $n)$, be an $n$-rectangular region which is a subset of $D$ and let $K$ be the set of points of $H$ which satisfy the equations $f_{1}\left(x_{1}, \cdots, x_{n}\right)$ $=0, \cdots, f_{r}\left(x_{1}, \cdots, x_{n}\right)=0$. For each point $p$ of $K$ let there exist an n-rectangular neighborhood of $p$ and an arrangement $j_{1}, \cdots, j_{n}$ of the indices $1,2, \cdots, n$ such that if $y_{i}=x_{j_{i}}$, $(i=1, \cdots, \quad n), \quad g_{m}\left(y_{1}, \cdots, \quad y_{n}\right)=f_{m}\left(x_{1}, \cdots, \quad x_{n}\right), \quad(m=1$, $2, \cdots, r)$, then the difference Jacobian 


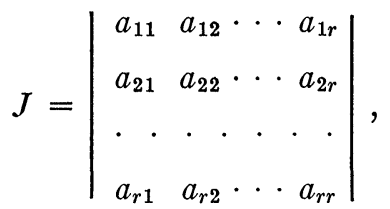

$$
\begin{aligned}
& \left\lfloor g_{i}\left(y_{1}, \cdots, y_{k-1}, y_{k}^{\prime}, y_{k+1}^{0}, \cdots, y_{r}^{0}, y_{r+1}, \cdots, y_{n}\right)\right. \\
& a_{i k}=\frac{\left.-g_{i}\left(y_{1}, \cdots, y_{k}, y_{k+1}^{0}, \cdots, y_{r}^{0}, y_{r+1}, \cdots, y_{n}\right)\right]}{\left[y_{k}^{\prime}-y_{k}\right]},
\end{aligned}
$$

as well as the elements of the principal diagonal and the minor obtained by deleting the first $i$ rows and the first $i$ columns, $(i=1$, $2, \cdots, r-1)$, are definite for all choices of the points in this neighborhood. Under these hypotheses, the set $K$ is an $(n-r)$ complex. Furthermore, if $K$ is interior to $H$, it is a finite set of closed $(n-r)$-manifolds.

Proof. An application of the Hedrick-Westfall implicit function theorem (loc. cit.) shows that $K$ consists of a finite collection $K_{1}, \cdots, K_{m}$ of maximal connected subsets each of which satisfies the hypotheses of Theorem 4. An application of Theorem 4 to each of these sets yields the fact that $K$ is an $(n-r)$-complex. In the case where $K$ is interior to $H$, Theorem 5 shows that $K_{1}, \cdots, K_{m}$ are closed $(n-r)$-manifolds.

In conclusion, it might be remarked that the hypotheses of Theorems 3 and 6 do not require the existence of derivatives of the functions that enter into these theorems.* On the other hand, the hypotheses of these theorems are met if the ordinary implicit function theorem hypotheses (involving derivatives of the first order) are valid. This omission of derivatives seems desirable since properties of analysis situs are not dependent upon derivatives. The assumption of continuity seems more essential since the transformations and correspondences of analysis situs are required to be continuous.

University of California at Los ANGeles

* An interesting example of a function that meets the hypotheses of these theorems and of the Hedrick-Westfall theorem but not those of the ordinary implicit function theorem, is obtained by making use of the well known example of a continuous, monotone, non-absolutely continuous function (see Hille and Tamarkin, American Mathematical Monthly, vol. 36 (1929), pp. 255-264). If $x$ is added to this function, an increasing function is obtained. The Hedrick-Westfall theorem applies directly to yield an inverse function $x=g(y)$. 